\title{
ESTUDIO DE LAS PROPIEDADES TERMO FISICAS DE CONGELACION DE MUSCULO DE CAMARON GIGANTE (Macrobrachium rosembergii): punto inicial de congelación y entalpia
}
F. Coronado *
J. O. Mendieta T.*
F. Kleeberg H. **

\section{RESUMEN}

El presente trabajo trata sobre la determinación del punto de congelación y entalpia de congelación del músculo de camarón gigante de Malasia (Macrobrachium rosembergii) cultivado en la Región San Martín.

El punto de congelación encontrado para un contenido de humedad de $78.6 \%$ del músculo, fue $-1.1{ }^{\circ} \mathrm{C}$, mientras que la entalpia de congelación alcanzó valores comprendidos entre -47.04 y $-70.44 \mathrm{kcal} / \mathrm{Kg}$.

Los valores obtenidos para el punto de congelación en función del contenido de humedad del músculo presentan buen grado de ajuste con los predichos por la ecuación de Schwartzberg. En cuanto a la entalpia de congelación, los valores obtenidos correlacionan bien con los predichos por las ecuaciones de Schwartzberg y Chen con Errores m los relativos de 1.36 y $5.84 \%$ respectivamente.

Palabras claves: Camarón gigante, punto de congelación, entalpia de congelación.

\section{INTRODUCCION}

El camarón gigante Macrobrachium rosembergii es un crustáceo natural de la región Indopacífica; es decir, del sur y sudeste asiático, partes de Oceanía y de algunas islas del Pacífico, New y Shingolka (New B. M. y Shingholka 1984).

Presenta una marcada diferencia, siendo los machos de mayor tamaño que las hembras.

Actualmente se cultiva con fines comerciales en muchos países como Honduras, Taiwán, Tailandia, Costa Rica, Israel, México y Filipinas.

Este recurso, de alto valor alimenticio y económico, ha sido introducido recientemente en la Región San Martín y se proyecta que, en un futuro cercano, alcanzará altos niveles de producción. En consecuencia, será necesario procesados

\footnotetext{
* Universidad Nacional de San Martín - Tarapoto. Fac. de Ingeniería Agroindustrial. Apartado 239 Tarapoto.

Universidad Nacional Agraria La Molina. Fac. de Ingeniería Pesquera. Lima
} 
con métodos apropiados que permitan mantener su calidad. Siendo la congelación el método de conservación más utilizado para el camarón, es de importancia conocer las propiedades termofísicas de congelación de éste.

Extensas investigaciones calorimétricas sobre la congelación de carne, pescado, frutas y vegetales se encuentran en la literatura especializada (Hsiech et al 1977), pero no se encuentra datos referidos al camarón gigante. Por esta razón, los objetivos del presente trabajo fueron:

- Determinar experimentalmente los parámetros termofísicos de congelación (punto inicial de congelación y entalpia del músculo de camarón gigante.

- Comparar los resultados experimentales de estos parámetros con los predichos por ecuaciones teóricas,

\section{MATERIALES Y METODOS}

El presente trabajo se llevó a cabo en las instalaciones del Laboratorio de Transformación del Departamento de Tecnología Pesquera de la Universidad Nacional Agraria La Molina, el Laboratorio de Análisis y Composición de Alimentos de la Universidad Nacional de San Martín, y en el laboratorios de la Empresa Agroindustrial DURHAN MAYO S.A.

La materia prima utilizada fue el camarón gigante de Malasia (Macrobrachiurn rosembergii).

\section{EQUIPOS Y MATERIALES}

- $\quad$ Balanza analítica Sauter, Tipo 414, aproximación, 0.1 mg., Alemania.

- Balanza eléctrica Sauter, Tipo S 1000T/0. 1, aproximación 0.1 g., Alemania.

- Congeladora Phillips, Modelo CCA-200, temperatura mínima - $15^{\circ}$ C, Lima - Perú.

- Equipo de destilación parnas Wagner

- Equipo digestor de proteínas

- Equipo extractor de grasa

- Estufa Memmert, Modelo TV 25V. rango de temperatura $30^{\circ} \mathrm{C}$ a $200^{\circ} \mathrm{C}$, Alemania Federal

- Garrafa térmica

- Mufla eléctrica Temco, Modelo 16303 , rango de temperatura $0^{\circ} \mathrm{C}$ a $120{ }^{\circ} \mathrm{C}$, Iowa - USA 
- Termómetro digital Lope, Modelo SP-625A, rango de temperatura $-199.9^{\circ} \mathrm{C}$ a 199. $9^{\circ} \mathrm{C}$, aproximación de $0.1 \mathrm{C}$, USA

- Termómetro Fisher Scientific, rango de temperatura $-10^{\circ} \mathrm{C}$ a $220^{\circ} \mathrm{C}$, aproximación $0.1^{\circ} \mathrm{c}$, USA

- Termopares cobre constantán No 24 acoplables.

Los materiales utilizados fueron diversos: balones, buretas, crisoles, embudos, erlenmeyers, fiolas, matraces, morteros, pipetas, placas petri y probetas.

Otros: poliuretano expandido (tecnopor), cuchillos, papel aluminio, hielo seco, regla graduada, ollas y sal.

\section{MÉTODOS}

\section{Análisis organoléptico}

Las características organolépticas de la materia prima fueron determinadas en base a las recomendaciones dadas por Barral et al (1986), Santos (1981) y New Singholka (1984).

\section{Análisis físico}

Los camarones enteros crudos fueron medidos y pesados. El rendimiento de la parte comestible, cabeza y residuos fueron calculados como un porcentaje del peso total.

\section{Análisis químico}

El análisis proximal de la parte comestible (humedad, proteína, grasa cruda y ceniza), fue realizado según los métodos de la A.O.A.C (1970).

\section{Preparación de muestras}

La preparación de las muestras, previa a la determinación del punto inicial de congelación y entalpia del músculo de camarón, se efectué según el Diagrama de Flujo mostrado en el gráfico 1.

Comprobada la frescura de la materia prima, fueron separadas las colas, eliminándose el caparazón, siendo seccionadas en trozos de aproximadamente 3 a $4 \mathrm{~cm}$. Con el objeto de contar con muestras de distintos grados de humedad, éstas 
fueron presecadas por medio de un ventilador, en intervalos de tiempo de 1 hora. Cada una de las muestras presecadas fueron envueltas en papel aluminio y mantenidas en refrigeración por 24 horas a fin de permitir la uniformización de la humedad de ellas.

\section{Determinación experimental del punto inicial de congelación}

Una muestra representativa de músculo de camarón conteniendo un termopar en su centro fue colocado en una garrafa térmica (gráfico 2) con aproximadamente $400 \mathrm{ml}$ de salmuera saturada a $-15^{\circ} \mathrm{C}$. La temperatura fue registrada cada 10 segundos hasta sobrepasar el punto inicial de congelación, el cual finalmente fue anotado. Luego se determiné la humedad de la muestra. Esta fue descongelada y congelada por 3 veces para verificar la reproductibilidad de la prueba.

Los valores experimentales fueron comparados con los obtenidos mediante una fórmula de correlación dada por Schwartzber (1976).

$$
\mathrm{t}_{1}=\frac{-\mathrm{En}_{2} \mathrm{RT}_{0}^{2}}{18 \mathrm{H}_{0}\left[1-\mathrm{N}_{\mathrm{s}}(1+\mathrm{b})\right]} \cdots \cdot[1]
$$

Donde $\mathrm{t}_{1}$ es numéricamente igual a la temperatura inicial de congelación expresada en ${ }^{\circ} \mathrm{C}$

\section{Determinación experimental de la entalpia de congelación}

Para la determinación experimental de la entalpia de congelación se usó el método de mezcla descrito por Kleeberg (1986).

A partir de los datos experimentales de masa y temperaturas y el correspondiente balance término del sistema, los valores de entalpia, fueron calculados, Alva (1990). La ecuación utilizada es:

$$
\mathrm{H}_{\mathrm{T}}=\frac{\left[-\mathrm{W}_{\mathrm{w}} \mathrm{C}_{\mathrm{w}}\left(\mathrm{T}_{\mathrm{wo}}-\mathrm{T}_{\mathrm{e}}\right)-\mathrm{W}_{\mathrm{m}} \mathrm{C}_{\mathrm{m}}\left(\mathrm{T}_{\mathrm{e}}-\mathrm{T}_{\mathrm{i}}\right)+\mathrm{q}_{\mathrm{c}}\right] \ldots . .[2]}{\mathrm{W}_{\mathrm{m}}} \ldots[
$$

Donde $\mathrm{H}_{\mathrm{T}}$ es entalpia de congelación $(\mathrm{Kcal} / \mathrm{kg}$ ). El significado de las demás variables se presenta en el Anexo 1.

El calor específico de la muestra $\left(\mathrm{C}_{\mathrm{m}}\right)$ por encima del punto de congelación fue calculado de la siguiente ecuación: 


$$
\mathrm{C}_{\mathrm{m}}=\mathrm{C}_{\mathrm{s}} \mathrm{n}_{\mathrm{s}}+\mathrm{C}_{\mathrm{s}} \mathrm{n}_{\mathrm{wo}} \quad \ldots . .[3]
$$

Donde $\mathrm{C}_{\text {s }}$ es el calor específico de $\left(\mathrm{kcal} / \mathrm{kg}{ }^{\circ} 2 \mathrm{C}\right)$ los sólidos totales. Este valor se obtuvo a partir de los calores específicos y fracciones de ¡nasa de los componentes de la fracción sólida de la muestra.

El valor del calor parasitario qc, calor asociado con la capacidad térmica del calorímetro entre las temperaturas inicial y final (Kcal), se determinó mediante un balance término sobre la garrafa térmica utilizada.

Con los valores de $\mathrm{q}_{\mathrm{c}}$ obtenidos y las correspondientes diferencias de temperaturas se trazó la curva mostrada en el gráfico 3. De esta forma, las posibles pérdidas de calor quedan incorporadas en $\mathrm{q}_{\mathrm{c}} \mathrm{y}$ explican la curvatura ascendente.

Los valores experimentales se los comparó con los obtenidos mediante fórmulas de correlación de Schwartzberg (1976) y Chen (1985b)

$$
\begin{aligned}
& \mathrm{H}=\quad\left(\mathrm{T}-\mathrm{T}_{1}\right)\left[\mathrm{C}_{\mathrm{f}}+\left(\mathrm{n}_{\mathrm{wo}}-\mathrm{bn}_{\mathrm{s}}\right) \frac{\mathrm{Ho}}{\left(\mathrm{T}_{\mathrm{o}}-\mathrm{T}\right)}\right] \ldots[4] \\
& \mathrm{H}=\quad\left(\mathrm{t}-\mathrm{t}_{\mathrm{i}}\right)\left(\mathrm{O} .37+\mathrm{O} .3 \mathrm{n}_{\mathrm{s}} \frac{+\mathrm{n}_{\mathrm{s}} \mathrm{RT}_{\mathrm{o}}}{\mathrm{Ms}_{\mathrm{s} \mathrm{tti}_{\mathrm{i}}}}\right.
\end{aligned}
$$

\section{Evaluación de los parámetros del punto de congelación}

\section{Fracción de masa de agua ligada " $b$ "}

Se la calculó mediante la ecuación [6], Schwartzberg H.G. (1976). Los valores de actividad de agua requeridos para la aplicación de la ecuación 161 se los determiné remplazando en la ecuación [7] del descenso crioscópico, las temperaturas iniciales de congelación, obtenidas experimentalmente.

$$
b=\frac{N_{1} A_{w 2}-N_{2} A_{w 1}+A_{w 1} A_{w 2}\left(N_{2}-N_{1}\right)}{A_{w 2}-A_{w 1}} \ldots . .[6]
$$




$$
\ln \mathrm{Aw}=\frac{\mathrm{M}_{\mathrm{w} 4} \mathrm{H}_{\mathrm{o}}}{\mathrm{R}} \frac{1}{\mathrm{~T}_{\mathrm{o}}} \frac{-1}{\mathrm{~T}}
$$

Relación de pesos moleculares "E”

Se lo obtuvo a partir del punto inicial de congelación de la muestra y la aplicación de la ecuación [8]. Schwartzberg, H.G. (1976)

$$
\mathrm{E}=(\mathrm{N}-\mathrm{b}) \frac{\left(1-\mathrm{A}_{\mathrm{w}}\right)}{\mathrm{A}_{\mathrm{w}}}
$$

Determinación del peso molecular efectivo “Ms"

El peso molecular efectivo "Ms" fue calculado mediante la ecuación 191 de Schwartzberg, H.G. (1976) y también con la ecuación [10] de Chen, C.S. 1985b, a partir de valores experimentales de entalpia dentro del rango de $10^{\circ} \mathrm{C}$ por debajo del punto inicial de congelación de las muestras.

$$
\begin{aligned}
& \mathrm{Ms}=18 / \mathrm{E} \ldots \ldots \ldots[9] \\
& \mathrm{Ms}_{\mathrm{s}}=\frac{\mathrm{n}_{\mathrm{s}} \mathrm{RT}_{0}^{2}}{\mathrm{ttr} \frac{\mathrm{H}_{\mathrm{f}}}{\mathrm{t}-\mathrm{tr}_{\mathrm{r}}}} 0.37-0.3 \mathrm{n}_{\mathrm{s}}
\end{aligned}
$$

\section{Evaluación de la exactitud}

Según Bizot (1983), citado por Alva J, M. 1990, el error medio relativo se define como la raíz cuadrada del error promedio, y fue calculado para expresar la exactitud o ajuste de los valores calculados con los obtenidos experimentalmente. La ecuación usada fue la siguiente: 


$$
\% \mathrm{R}=\mathrm{i}_{\mathrm{i}=1}^{\mathrm{n}} \sum \quad\left[\left(\mathrm{V}_{\exp }-\mathrm{V}_{\mathrm{c}}\right) / \mathrm{V}_{\exp }\right] \mathrm{n} \times 100 \quad \ldots . .[19]
$$

Donde $\mathrm{n}$ es el número de puntos experimentales, $\mathrm{V}_{\exp }$ es el valor obtenido experimentalmente, $\mathrm{V}_{\mathrm{c}}$ el valor calculado mediante la ecuación respectiva.

\section{RESULTADOS Y DISCUSION}

\section{ANALISIS ORGANOLÉPTICO}

La materia prima utilizada presentó las siguientes características:

- Color azul verdoso y brillante con quelípedos (pinzas) azules, propio del camarón en buenas condiciones de calidad.

- Olor característico, muy suave, a agua dulce.

- Textura flexible y muy firme.

La percepción de las características anteriormente mencionadas indicaron que la materia prima utilizada fue de muy buena calidad.

Las características físicas del camarón gigante utilizado fueron:

- $\quad$ Longitud promedio

- Peso entero

- Fracción de peso limpio

- Fracción de peso residuos
$: 16.76 \mathrm{~cm}$

$: 49.16 \mathrm{~g}$.

$: 0.3542$

$: 0.6458$

\section{ANALISIS QUÍMICO}

La composición química del camarón gigante se presenta en el cuadro 1 . Puede apreciarse que se trata de una especie con alto contenido de humedad y proteína. El porcentaje de estos componentes, y los obtenidos para ceniza y carbohidratos, son comparables a los valores que para camarones de agua dulce informan Wheaton y Lawson (1985). El contenido graso hallado resulta bajo si se lo compara con el valor encontrado por Sidwell (1977), citado por Reddy et al (1981), pero está dentro del rango de valores determinados por Wheaton y Lawson (1985). 


\begin{tabular}{lc} 
Cuadro 1 \\
COMPOSICIÓN QUÍMICO PROXIMAL DE MÚSCULO DE CAMARÓN \\
DE AGUA DULCE \\
(M. rosenbergii) \\
\hline Componente & Porcentaje (\%) \\
\hline Humedad & 78.6 \\
Proteína & 19.4 \\
Grasa & 0.5 \\
Ceniza & 1.2 \\
Carbohidratos & 0.3
\end{tabular}

\section{PUNTO INICIAL DE CONGELACIÓN Y PARÁMETROS EMPÍRICOS}

Los valores del punto inicial de congelación para músculo de camarón gigante, en función al contenido de humedad, son presentados en el cuadro 2 . Puede apreciarse que el punto inicial de congelación aumenta al disminuir el contenido de humedad. Este comportamiento se informa para otros productos similares $(4,5,8,11,12)$.

Los valores obtenidos para los parámetros empíricos de congelación de músculo de camarón de agua dulce fueron los siguientes:

Constante empírica, $\mathrm{b}=0.29$

Peso molecular efectivo, $\mathrm{Ms}=492.07$

Constante empírica, $\mathrm{E}=0.03658$

El valor de b calculado para el camarón de agua dulce (Macrobrachium rosenbergii) representa la fracción de agua no congelable/Kg. de materia seca, el que es cercano al valor que informa Kleeberg, H.G. (1986) para pescado crudo y cocido, sin embargo resulta superior al rango de valores indicados por Duckworth (1971), mencionado por Gruda y Postolski (1986), que es de 0.24 a 0.27.

El peso molecular efectivo, M, encontrado para el material en estudio es inferior al que para productos similares, como caracol fresco, informa Alva (1990), y pescado crudo, Kleeberg (1986). Esto se debe al contenido de humedad de camarón fresco que es más elevado que el de los productos mencionados, puesto que el peso molecular efectivo varía en forma inversa con el contenido de humedad.

De otro lado, el valor de E encontrado para el camarón de agua dulce presentará una tendencia creciente similar a los valores de $\mathrm{E}$ que, informa Kleeberg (1986), para pescado crudo y cocido, dado que E está en relación inversa al peso molecular $(\mathrm{M}$,$) .$ 
Los valores de punto inicial de congelación fueron calculados también mediante la ecuación [1] propuesta por Schwartzberg (1981), la cual utiliza los parámetros empíricos mencionados anteriormente y presentados en el cuadro 2 . El error medio relativo (EMR) estimado es de $6.38 \%$ lo que indica una buena concordancia de los valores experimentales con los predichos mediante la ecuación propuesta por Schwartzberg (1976). En el gráfico 4 se presenta los valores experimentales y calculados para el punto inicial de congelación del músculo de camarón gigante.

\section{Cuadro 2}

PUNTO DE CONGELACIÓN EXPERIMENTAL Y CALCULADO DE CARNE DE CAMARÓN GIGANTE

Humedad Sólidos totales Punto inicial de congelación

100nwo ns $(\mathrm{kg} / \mathrm{kg})$

Exp. $\mathrm{T}_{\mathrm{i}}\left({ }^{\circ} \mathrm{C}\right)$

Calc.

\begin{tabular}{llll}
78,6 & 0.214 & -1.1 & -1.11 \\
76.0 & 0.240 & -1.3 & -1.60 \\
72.5 & 0.275 & -1.6 & -1.60 \\
70.4 & 0.296 & -1.8 & -1.80 \\
65.9 & 0.341 & -2.3 & -2.29 \\
54.0 & 0.360 & -2.5 & -2.53 \\
\hline
\end{tabular}

ERROR (EMR)

6,38

\section{ENTALPIA DE CONGELACION}

Los resultados experimentales de entalpia de congelación obtenidos para músculo de camarón, se los presenta en el cuadro 3. Estos valores muestran buena concordancia con los calculados mediante las ecuaciones de Schwartzberg (1976) y Chen (1985h), siendo ¡os valores del Error Medio Relativo de 1.36 y 5.84\%, respectivamente. Estas desviaciones son aceptables considerando las condiciones de trabajo y la sencillez de los equipos utilizados. El gráfico 5, ilustra la concordancia de los resultados experimentales con los teóricos, especialmente con la ecuación de Schwartzberg (1981). 
Al comparar estos resultados con los obtenidos para caracol crudo, Alva (1991), puede notarse que son más elevados para la misma temperatura. Esto es debido a que a mayor contenido de humedad, mayores son los valores de entalpia. Efecto similar observó Riedel (1956), citado por Alva (1990), trabajando con muestras de pescado de diferente contenido de humedad.

\section{Cuadro 3}

ENTALPIA DE CONGELACIÓN EXPERIMENTAL Y CALCULADO PARA CAMARÓN GIGANTE

\begin{tabular}{|c|c|c|c|}
\hline \multirow{3}{*}{$\mathrm{T}^{\circ} \mathrm{C}$} & \multicolumn{3}{|c|}{ Entalpia (Kcal/Kg) } \\
\hline & \multirow[t]{2}{*}{ Experimental } & \multicolumn{2}{|c|}{ Calculado } \\
\hline & & SCHW. ${ }^{1}$ & $\mathrm{CHEN}^{2}$ \\
\hline 8.0 & --- & 7.86 & 7.86 \\
\hline 5.0 & --- & 5.27 & 5.27 \\
\hline 3.0 & --- & 3.54 & 3.54 \\
\hline 1.0 & --- & 1.81 & 1.81 \\
\hline-1.1 & 0.00 & 0.00 & 0.00 \\
\hline-5.0 & - 47.04 & $-47,01$ & -47.41 \\
\hline-9.8 & -55.67 & -55.65 & -55.81 \\
\hline-11.0 & -57.08 & -55.65 & -55.81 \\
\hline-15.6 & -60.87 & -60.97 & -60.77 \\
\hline-17.5 & -62.38 & -62.37 & -62.04 \\
\hline-22.8 & -65.85 & -65.87 & -65.20 \\
\hline-30.5 & -70.44 & -70.44 & -69.26 \\
\hline \multicolumn{2}{|c|}{ ERROR (EMR) } & $1.36 \%$ & $5.84 \%$ \\
\hline
\end{tabular}

\section{CONCLUSIONES}

Las principales conclusiones de este trabajo son las siguientes:

- El punto de congelación del músculo de camarón gigante es $-1.1^{\circ} \mathrm{C}$ para un contenido de humedad de $78.6 \%$. 
- El valor de la entalpia de congelación varía de $-47.04 \mathrm{Kcal} / \mathrm{Kg}$ a $70.44 \mathrm{Kcal} / \mathrm{Kg}$.

- $\quad$ El peso molecular efectivo tiene un valor de $492.07 \mathrm{Kg} / \mathrm{Kg}$ - mol.

- Los valores del punto de congelación de camarón gigante correlacionan bien con el contenido de sólidos y se ajustan a la ecuación propuesta por Schwartzber (1976).

Los parámetros que definen la cualidad de ajuste son:

$$
\mathrm{b}=0.29 \quad \mathrm{E}=0.03658
$$

- La ecuación [4] de Schwartzberg (1976), y la ecuación [5] de Chen (1985b), correlacionan bien los datos experimentales de entalpia de congelación, con EMR inferiores a $1.36 \%$ y $5.84 \%$ respectivamente.

\section{BIBLIOGRAFIA}

ALVA JORGE, M. 1990. Estudio de los parámetros termodinámicos de congelamiento del caracol (Thais chocolata) precocido". Tesis UNALM. Lima-Perú.

A,I.C. 1970. Official Methods of Analysis Association of Official Agriculture Chemist., $11^{\text {ava }}$ Edición.

BARRAL A.O., BERGAMASHI, H.J. y C.A. CASTANON 1986. "Planillas de puntaje organoléptico para langostino (Pleoticus muelleri) fresco y congelado". FAO Informe de pesca $\mathrm{N}^{0}$ 421. Suplemento. Santiago de Chile.

CHEN, C.S. 1985a. "Thermodynamic analysis of the freezing and thawing of foods: Entahalpy and Aarent Specific Heat. J. Food Sci”, 50: 1158 1162,

CHEN, C.S. 1985B. "Thermodynamic analysis of the freezing and thawing of foods: Ice content and Mollier Diagram. J. Food Sci”. 50: 1163- 1166.

GRUDA, Z. y 1. POSTOLSKI 1986. Tecnología de la congelación de los alimentos. Edit. Acribia S.A. 631.

HSIEH., L.E. LEREW, D.R. HELDMAN 1977. Prediction of freezing times for foods as influeced by product properties. J. Food Process Engineering. Vol. I 183. 
KLEEBERG, H.F. 1986. Estudio comparativo dos parámetros termodinámicos e da cinetica de congelamiento de file de pescado (Cynoscion petranas) cru e cozido". Tesis. Universidade de Gradual de Campinas. Sao Paulo.

NEW, G.M. y S. SINGHOLKA 1984. Cultivo de camarón de agua dulce. Manual para el cultivo de Macrobrachium rosenbergii Documento técnico de pesca (225): 118 pag.

REDDY, KW. NIP, and S. TANG 1981. Changes in fatty acids and sensory qualitv of fresh water prawn (Macrobrachium rosenbergii) sotred under frozen conditions. J. Food Sci. 46: 353-356.

SANTOS, M.R. 1986. "Manual de procesamiento y control de calidad del langostino congelado. Planta Piloto Dpto. de Congelado". No 2 Año II Comisión Editorial Publicaciones. Callao - Perú.

SCHWARTZBERG, H.G. 1976. "Effective heat capacities for the freezing and thawing of food. J. Food Sci”. 41: 152-156.

SCHWARTZBERG, HG. 1981. "Mathematocal Analysis of the Freezing and Thawing of Foods". AICHE. Summer Meeting, August Detroit. USA.

WHEATON, F. and T. LAWSON 1985. "Processing Aquatic Food Products" Ed. Jhohn Wiley \& Sons. USA 


\section{ANEXO 1 \\ NOMENCLATURA UTILIZADA}

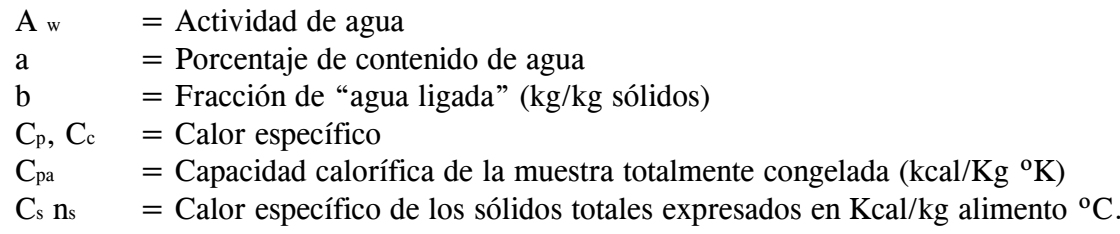

$\mathrm{C}_{\mathrm{s}}, \mathrm{C}_{\mathrm{w}}, \mathrm{C}_{\mathrm{w}(\mathrm{t})}$,

$\mathrm{C}_{\mathrm{n}(\mathrm{t})} \quad=$ Calor específico de la porción sólida, del agua, del agua no congelada a del agua congelada del alimento, que puede no ser dependiente de la temperatura si el calor específico del hielo es constante en el rango de temperatura del producto, respectivamente $\left(\mathrm{kcal} / \mathrm{Kg}{ }^{\circ} \mathrm{C}\right)$

$\mathrm{C} \mathrm{m}=$ Calor asociado de la muestra (Kcall $/ \mathrm{Kg}$ alimento $\left.{ }^{\circ} \mathrm{C}\right)$

$\mathrm{E} \quad=$ Constante empírica

$\mathrm{H} \quad=$ Entalpia de congelación $(\mathrm{Kcal} / \mathrm{Kg})$

Ho $\quad=$ Calor latente de fusión del agua $(79.8 \mathrm{Kcal} / \mathrm{Kg})$

$\mathrm{H}_{\mathrm{f}} \quad=$ Entalpia por debajo de la congelación $(\mathrm{Kcal} / \mathrm{Kg})$

$\mathrm{h}=$ Contenido de hielo (\%)

$\mathrm{M}_{\mathrm{w}} \quad=$ Peso molecular del agua pura $(18 \mathrm{Kg} / \mathrm{Kg}-\mathrm{mol})$

n $\quad=$ Fracción de agua no congelada a la temperatura de congelación $(\mathrm{Kg} / \mathrm{Kg}$ de alimento).

$\mathrm{n}_{\mathrm{s}} \quad=$ Fracción de sólidos totales (solubles e insolubles, $\mathrm{Kg} / \mathrm{Kg}$ de alimento)

$\mathrm{n}_{\text {wo }} \quad=$ Fracción inicial de agua al alimento $(\mathrm{Kg} / \mathrm{Kg}$ alimento $)$

$\mathrm{n}_{\mathrm{h}} \quad=$ Fracción de hielo $(\mathrm{Kg} / \mathrm{Kg}$ de alimento)

$\mathrm{q}_{\mathrm{c}}=$ Calor asociado con la capacidad térmica del calorímetro entre las temperaturas inicial y final (Kcal)

$\mathrm{R}=$ Error medio relativo

$\mathrm{S} \quad=$ Velocidad de formación de hielo $\left(\mathrm{Kg} / \mathrm{Kg}\right.$ de al. $\left.{ }^{\circ} \mathrm{C}\right)$ ó $\mathrm{Kg}$ de masa de hielo formado por grados de descenso en la temperatura por $\mathrm{Kg}$ de alimento.

T. $\mathrm{t}=$ Temperatura a temperatura de congelación.

To $\quad=$ Temperatura del punto de congelación del agua $\left(273.15^{\circ} \mathrm{K}\right)$

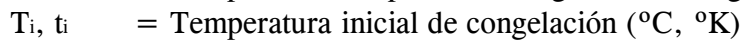

$\operatorname{tr} \quad=$ Temperatura de referencia

two $\quad=$ Temperatura inicial al agua $\left({ }^{\circ} \mathrm{C}\right)$

$\mathrm{T}_{\mathrm{e}} \quad=$ Temperatura de equilibrio

$\mathrm{W}_{\mathrm{w}} \quad=$ Peso del agua

$\mathrm{W}_{\mathrm{m}} \quad=$ Peso de la muestra del camarón gigante $(\mathrm{Kg}$.

$0.20=$ Base arbitraria del calor específico de los constituyentes sólidos. 


\section{Grafico 1}

GARRAFA TERMICA UTILIZADA EN LA DETERMINACION DE PUNTO DE CONGELACION DE MUSCULO DE CAMARON

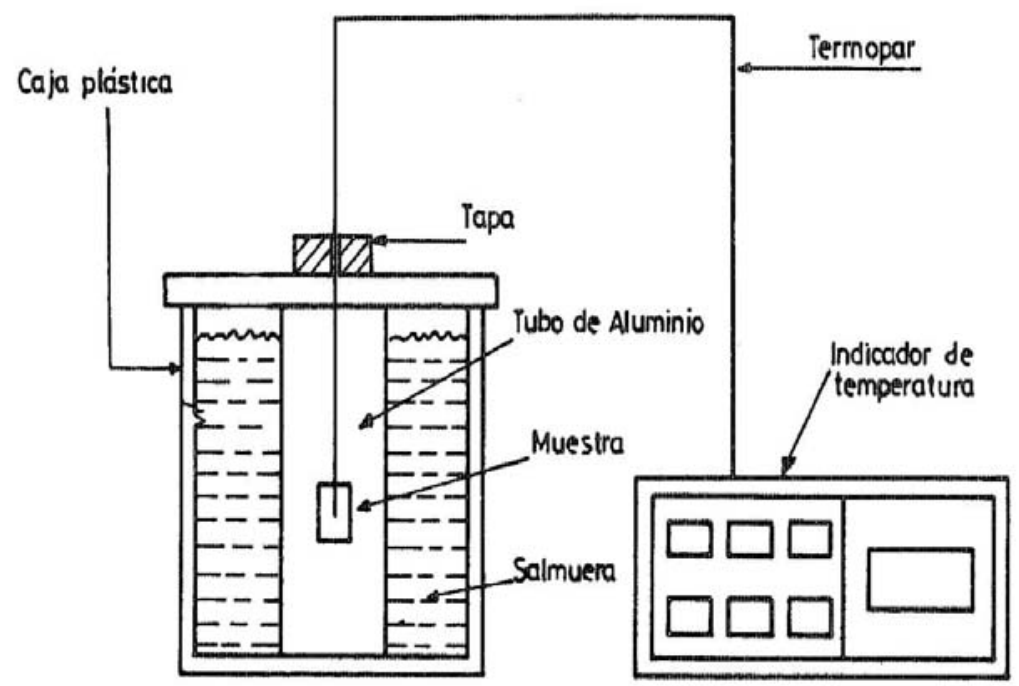


Grafico 2

CURVA DE CALIBRACION DE LA GARRAFA TERMICA

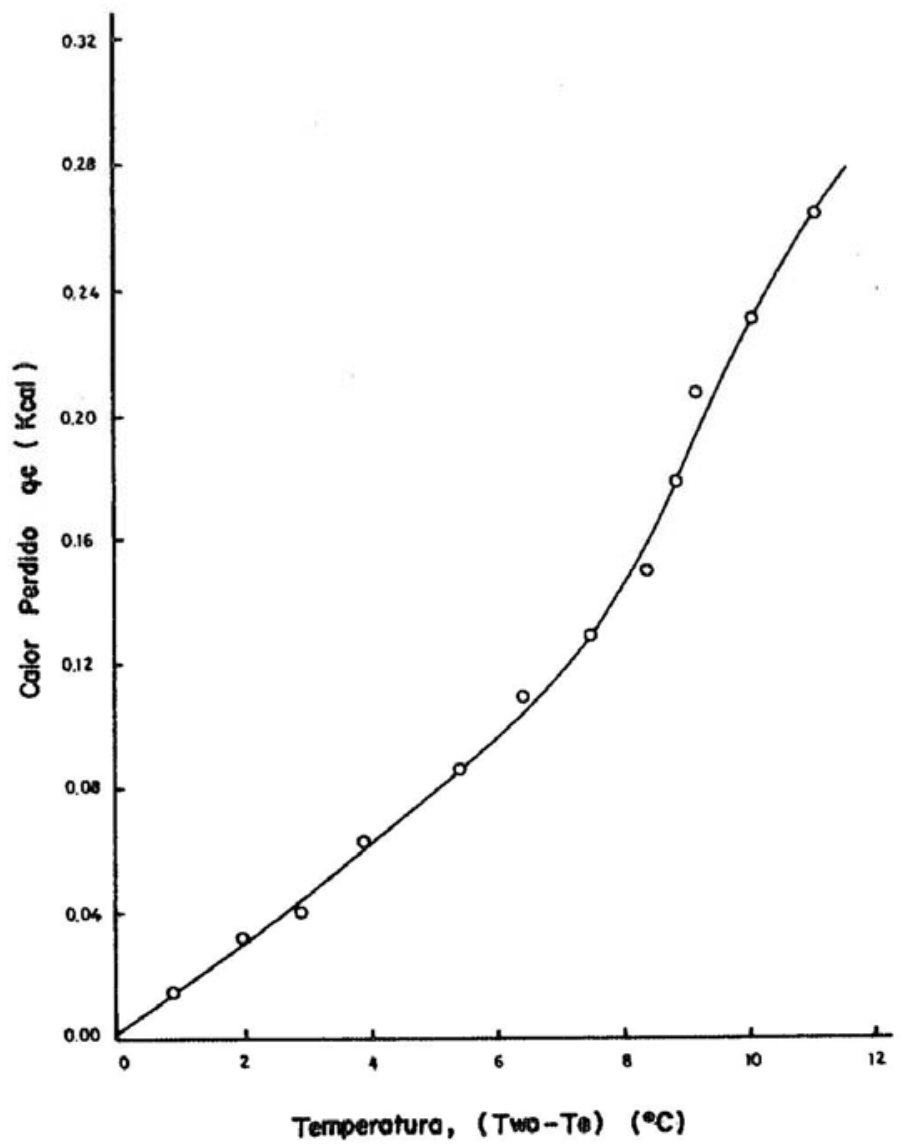




\section{Grafico 3}

CURVA TEORICA DEL PUNTO DE CONGELACION DE MUSCULO DE CAMARON GIGANTE EN COMPARACION CON LOS RESULTADOS EXPERIMENTADOS

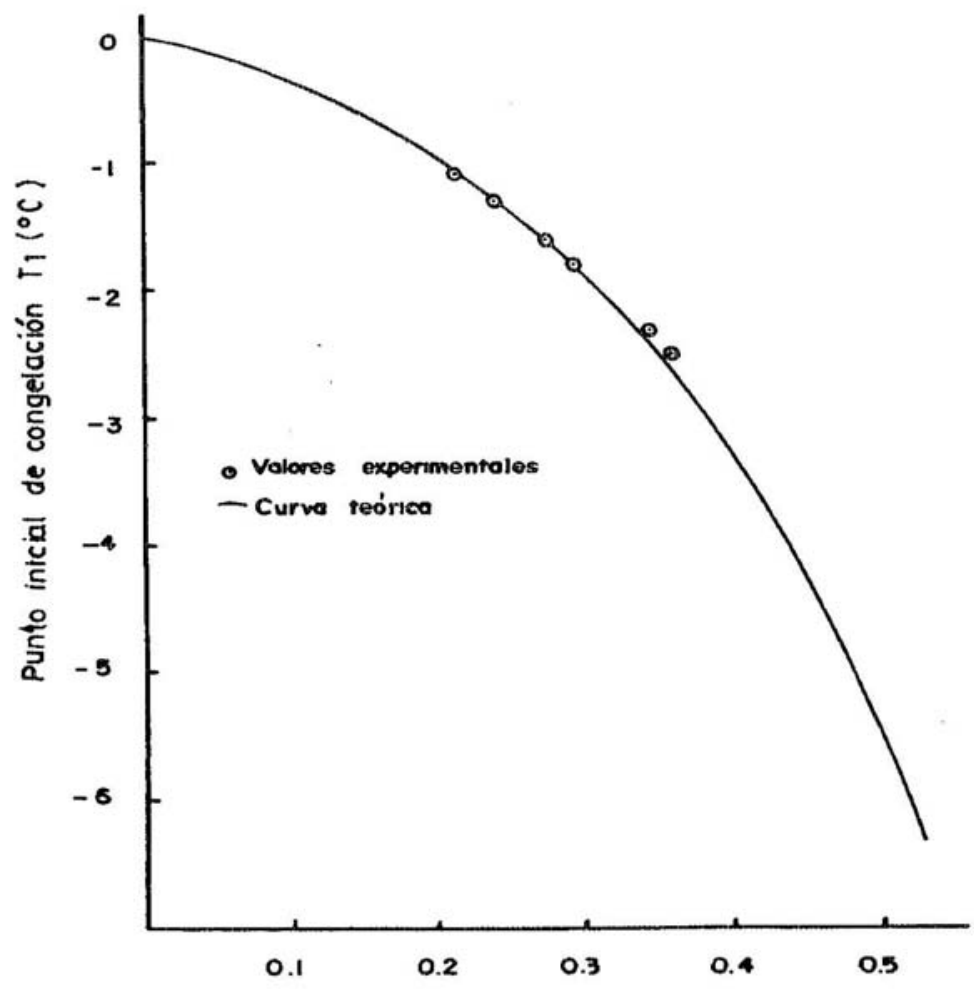

Fracción de sólidos ns ( $\mathrm{kg}$ ) 


\section{Grafico 4}

COMPARACION DE VALORES EXPERIMENTALES DE ENTALPIA CON TEORICOS OBTENIDOS MEDIANTE LA ECUACION DE SHWARTZBERG

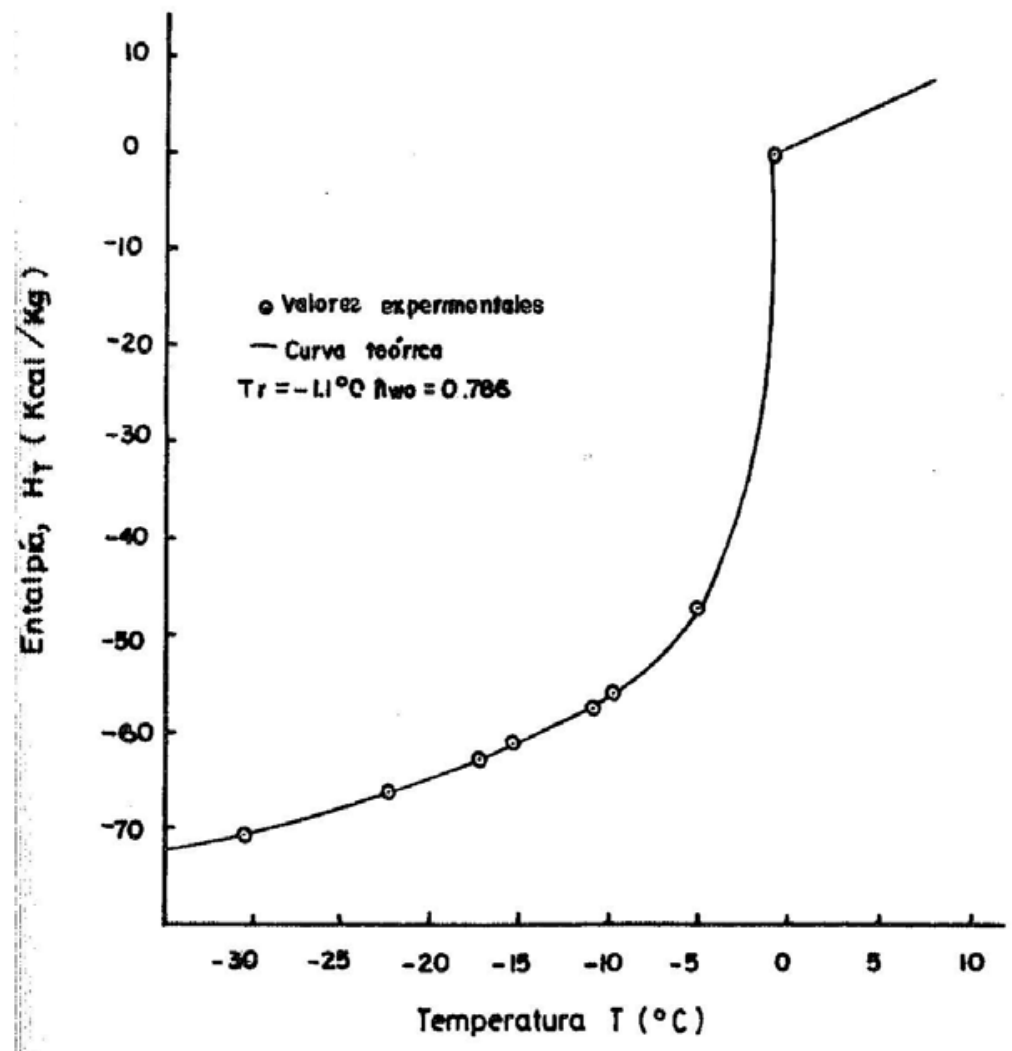

